\title{
Using poly(lactic-co-glycolic acid) microspheres to encapsulate plasmid of bone morphogenetic protein 2/polyethylenimine nanoparticles to promote bone formation in vitro and in vivo
}

\author{
This article was published in the following Dove Press journal: \\ International Journal of Nanomedicine \\ 9 August 2013 \\ Number of times this article has been viewed
}

\author{
Chunyan Qiaol,* \\ Kai Zhang ${ }^{2, *}$ \\ Han Jin' \\ Leiying $\mathrm{MiaO}^{3}$ \\ Ce Shi' \\ Xia Liu' \\ Anliang Yuan' \\ Jinzhong Liu' \\ Daowei $\mathrm{Li}^{\prime}$ \\ Changyu Zheng ${ }^{4}$ \\ Guirong Zhang \\ Xiangwei Li' \\ Bai Yang ${ }^{2}$ \\ Hongchen Sun' \\ 'Department of Pathology, School of \\ Stomatology, Jilin University, Changchun, \\ ${ }^{2}$ State Key Laboratory of Supramolecular \\ Structure and Materials, College of \\ Chemistry, Jilin University, Changchun, \\ ${ }^{3}$ Institute and Hospital of Stomatology, \\ Nanjing University Medical School, \\ Nanjing, People's Republic of China; \\ ${ }^{4}$ Molecular Physiology and Therapeutics \\ Branch, National Institute of Dental and \\ Craniofacial Research, National Institutes \\ of Health, Bethesda, MD, USA; ${ }^{5}$ Department \\ of Biochemistry, School of Basic Medicine, \\ Jilin University, Changchun, People's \\ Republic of China \\ *These authors contributed equally to this \\ work
}

Correspondence: Hongchen Sun Department of Pathology, School of Stomatology, Jilin University, Changchun, I3002 I, People's Republic of China

Tel +8643188796012

Fax +8643188975348

Email hcsun@jlu.edu.cn

\begin{abstract}
Repair of large bone defects is a major challenge, requiring sustained stimulation to continually promote bone formation locally. Bone morphogenetic protein 2 (BMP-2) plays an important role in bone development. In an attempt to overcome this difficulty of bone repair, we created a delivery system to slowly release human BMP-2 cDNA plasmid locally, efficiently transfecting local target cells and secreting functional human BMP-2 protein. For transfection, we used polyethylenimine (PEI) to create $\mathrm{pBMP}-2 / \mathrm{PEI}$ nanoparticles, and to ensure slow release we used poly(lactic-co-glycolic acid) (PLGA) to create microsphere encapsulated pBMP-2/ PEI nanoparticles, PLGA@pBMP-2/PEI. We demonstrated that pBMP-2/PEI nanoparticles could slowly release from the PLGA@pBMP-2/PEI microspheres for a long period of time. The 3-15 $\mu \mathrm{m}$ diameter of the PLGA@pBMP-2/PEI further supported this slow release ability of the PLGA@pBMP-2/PEI. In vitro transfection assays demonstrated that pBMP-2/PEI released from PLGA@pBMP-2/PEI could efficiently transfect MC3T3-E1 cells, causing MC3T3E1 cells to secrete human BMP-2 protein, increase calcium deposition and gene expressions of alkaline phosphatase $(A L P)$, runt-related transcription factor 2 (RUNX2), SP7 and I type collagen (COLL I), and finally induce MC3T3-E1 cell differentiation. Importantly, in vivo data from micro-computed tomography (micro-CT) and histological staining demonstrated that the human BMP-2 released from PLGA@pBMP-2/PEI had a long-term effect locally and efficiently promoted bone formation in the bone defect area compared to control animals. All our data suggest that our PLGA-nanoparticle delivery system efficiently and functionally delivers the human BMP-2 cDNA and has potential clinical application in the future after further modification.
\end{abstract}

Keywords: gene therapy, bone regeneration, biodegradable polymer, human BMP-2

\section{Introduction}

A clinician continues to face the challenge of repairing bone defects in daily practice. Bone grafting is a common treatment for bone defects, but the long procedure of grafting, donor tissue availability, and donor site morbidity, limits treatment ${ }^{1}$ and highlights the need for research into new methods. Bone morphogenetic protein 2 (BMP-2) is one of the strongest osteoinductive proteins and plays an important role in the development of bone and cartilage. ${ }^{2}$ Many studies have used BMP-2 protein to stimulate bone formation in vitro and in vivo. ${ }^{3,4}$ However, recombinant BMP-2 protein is expensive and rapidly degrades in vivo. ${ }^{5,6}$ Gene therapy may provide an alternative method for delivery of BMP-2. ${ }^{6-11}$ 
Gene therapy can be divided into two major vector systems that can be used to deliver the therapeutic gene into target cells: viral and non-viral vector systems. Currently, viral vectors are still the most efficient vectors. Viral vectors, however, can induce many unwanted side effects compared to non-viral vectors. Recently, nanotechnology provided a useful tool to create a novel non-viral vector system. ${ }^{12}$ Polyethylenimine (PEI) is a promising candidate for delivering negatively charged DNA in vitro and in vivo because PEI has a high positive charge density in aqueous solutions. PEI forms complexes with DNA via cooperative electrostatic interactions between ammonium groups of polycation and phosphate groups of the DNA. ${ }^{13}$ PEI in the PEI/DNA complexes displays favorable characteristics of DNA protection, cell binding and uptake, endosomal escape, and release from the carrier. ${ }^{14,15}$

Effective BMP-2 treatment for bone defects locally requires sustained BMP-2 for a sufficient period of time to allow osteoprogenitor cells to migrate to the target site and differentiate into osteoblasts. ${ }^{16-19}$ Animal studies have demonstrated that BMP-2 proteins in collagen powder or sponges quickly release and are only retained for a very short period of time after implantation. ${ }^{16,20}$ Entrapment of bioactive factors using biodegradable polymer material is a promising tool to control the release of drugs, proteins, and DNA. A synthetic material, poly(lactic-co-glycolic acid) (PLGA) is a wellcharacterized biodegradable material for controlled release systems. Previously, BMP-2 protein entrapped in PLGA microspheres had been shown to release therapeutic proteins to stimulate osteogenesis. ${ }^{16}$ To create an effective non-viral delivery system, we used PEI to create nanoparticles of pBMP-2/ PEI plasmid DNA in the present study. To achieve long-term controllable release of pBMP-2/PEI nanoparticles, we used PLGA to entrap pBMP-2/PEI nanoparticles in order to effectively and efficiently promote bone formation locally.

\section{Materials and methods Construction of plasmid and PBMP-2/PEI nanoparticle preparation}

To construct pBMP-2, cDNA (1547 base pairs) of BMP-2 in pCMV6-XL4 (Origene Technologies, Rockville, MD, USA) was digested with Not I, filled in with T4 DNA polymerase, and then digested with Eco RI. Expression vector pACCMVpLpA was digested with Bam HI, filled in with T4 DNA polymerase, and then digested with Eco RI. The treated BMP2 cDNA was ligated with treated pACCMV-pLpA vector to get $\mathrm{pBMP}-2$. Human CMV promoter was used to drive human BMP-2 cDNA.
PEI (molecular weight $=25,000$ Da, BPEI25k (Branched PEI 25k), Sigma-Aldrich, St Louis, MO, USA) was suspended in sterile water at $2 \mathrm{mg} / \mathrm{mL}$ and adjusted to $\mathrm{pH} 7.4$. pBMP-2/PEI formulation was prepared with PEI nitrogen to DNA phosphate $(\mathrm{N} / \mathrm{P})$ at a molar ratio of $8: 1$. Complexes were formed by slowly adding pBMP-2 to PEI solution and gently mixed by pipetting up and down 5-8 times. Complexes were incubated at room temperature for 20 minutes prior to use. The average hydrodynamic diameter and the zeta potential of the nanoparticles were determined by dynamic light scattering (Malvern Zetasizer Nano Z; Malvern Instruments, Malvern, UK) at room temperature.

\section{Preparation of PLGA@pBMP-2/PEI microspheres}

PLGA (molecular weight 40,000 Da, Sigma-Aldrich, St Louis, MO, USA) microspheres loaded with pBMP-2/PEI nanoparticles were prepared via the water-oil-water emulsion solvent evaporation method. Briefly, $0.6 \mathrm{~mL}$ of pBMP-2/ PEI solution containing $36 \mu \mathrm{g}$ of pBMP-2 was emulsified in $2 \mathrm{~mL}$ of methylene chloride (dichloromethane, 99.8\%, Sigma-Aldrich, St Louis, MO, USA) containing 2\% of PLGA (wt/vol) by homogenization (homogenizer 985370-XL biospec) at 5000 revolutions per minute (rpm) in an ice bath for 10 seconds to form the primary emulsion (water/oil [W/O]) (Figure 1). Thereafter, the primary emulsion was poured into $10 \mathrm{~mL}$ of poly(vinyl alcohol) (PVA, Sigma-Aldrich) aqueous solution $(3.0 \%, \mathrm{wt} / \mathrm{vol})$ and homogenized at $10,000 \mathrm{rpm}$ in an ice bath for 5 seconds (Figure 1). The double emulsion (water-oil-water) was diluted in $20 \mathrm{~mL}$ of polyvinyl alcohol (PVA) solution $(0.5 \%, \mathrm{wt} / \mathrm{vol})$ under moderate magnetic stirring at room temperature for 2-3 hours to evaporate the organic solvent. Finally, formed microspheres were collected by centrifugation at 5,000 rpm for 15 minutes, washed three times with distilled water and freeze-dried using a lyophilizer (ALPHA 1-2 LD, Laurel, MD, USA) to sublimate remaining water and obtain PLGA microspheres (Figure 1). The dried microspheres were stored at $-20^{\circ} \mathrm{C}$ until use. Control PLGA microspheres and fluorescein isothiocyanate (FITC) (SigmaAldrich, St Louis, MO, USA)-labeled PLGA microspheres were prepared using the same protocol described above, but replacing the $\mathrm{pBMP}-2 / \mathrm{PEI}$ complex by $\mathrm{PEI}$ only or $\mathrm{pBMP}-2 /$ PEI/FITC.

\section{Preparation of gelatin sponge loaded PLGA@PBMP-2/PEI}

$0.58 \mathrm{mg}$ of PLGA@pBMP-2/PEI containing $10 \mu \mathrm{g}$ of pBMP-2 were dispersed in water at high density, and added 


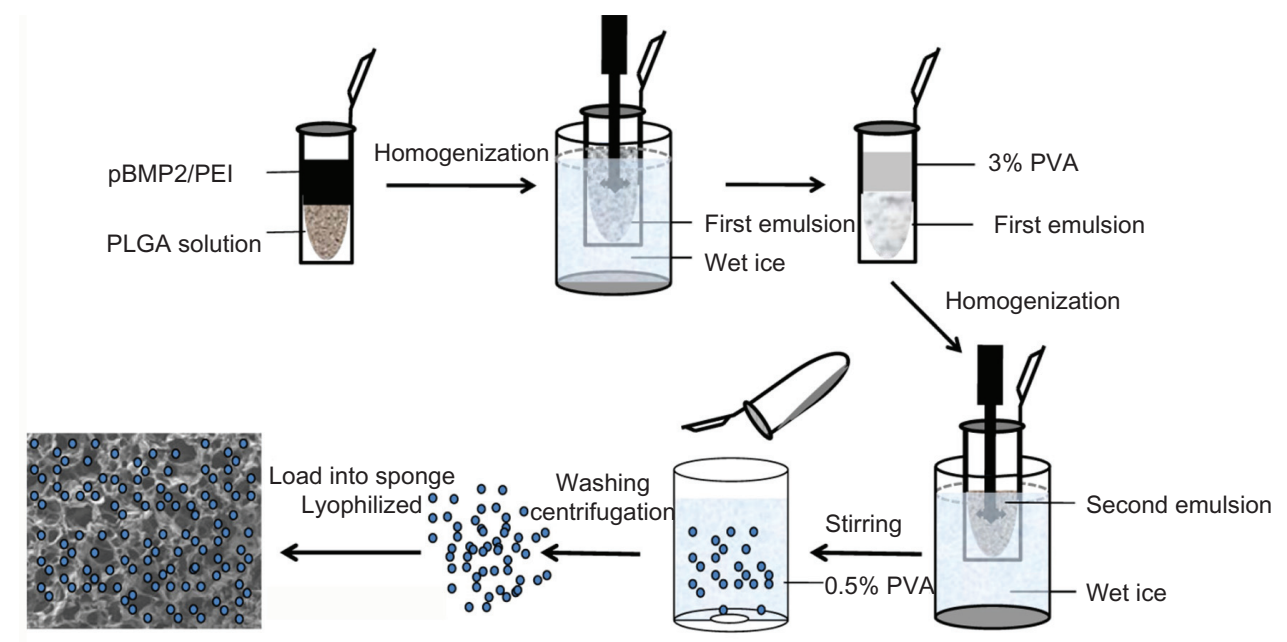

Figure I Diagram of preparation of gelatin sponge loaded with PLGA@pBMP-2/PEl.

Abbreviations: pBMP2/PEI, plasmid of bone morphogenetic protein 2/polyethlenimine; PLGA, poly(lactic-co-glycolic acid); PVA, polyvinyl alcohol.

onto the gelatin sponge; the gelatin sponge was lyophilized after the mixture was absorbed into it (Figure 1).

\section{Characteristics of PLGA@pBMP-2/PEI}

\section{Particle morphology}

Particle morphology of lyophilized microspheres was observed using field emission scanning electron microscopy (FESEM6700F, JEOL, Tokyo, Japan). Fluorescent images were acquired using a fluorescence microscope.

\section{Entrapment and drug loading rate of PLGA@pBMP-2/PEI}

PLGA entrapment efficiency of pBMP-2/PEI was evaluated by measuring unbound pBMP-2 in the solution. Supernatant in the PLGA@pBMP-2/PEI solution was recovered by centrifugation at 5,000 rpm for 15 minutes. Free pBMP-2 concentration in the supernatant was determined using a spectrophotofluorometer (RF-1501, Shimadzu, Kyoto, Japan) with a Quant-It ${ }^{\mathrm{TM}}$ PicoGreen dsDNA quantitation assay reagent (Life Technologies, Grand Island, NY, USA). ${ }^{21}$ Encapsulation efficiency was defined as percent of pBMP-2 encapsulated in $\mathrm{pBMP}-2 / \mathrm{PEI}$ by PLGA with respect to initial amount of pBMP-2. Drug loading rate (wt $\%$ ) was the percent of pBMP-2 entrapped in the microspheres in the total amount of PLGA@pBMP-2/PEI. ${ }^{22}$ The amount of pBMP-2 in the microspheres was determined by subtracting the amount of pBMP-2 recovered in the wash solutions from the initial amount of pBMP-2 added.

\section{In vitro release assay}

Twentymg PLGA@pBMP-2/PEI was incubated in $1 \mathrm{~mL}$ of phosphate buffered saline (PBS) $(\mathrm{pH} 7.4)$ in a rotary shaker at $30 \mathrm{rpm}$ and $37^{\circ} \mathrm{C}$. Supernatants were collected after spinning at 10,000 rpm for 5 minutes at days 1, 3, 7, $10,15,17,20,24,28,30,33,35,40$, and 45 . At each time point, the microspheres were resuspended in $1 \mathrm{~mL}$ of fresh PBS. Amount of DNA in the supernatant was determined with a Quant-It ${ }^{\mathrm{TM}}$ PicoGreen dsDNA quantitation assay reagent (Life Technologies) using a spectrophotofluorometer (RF-1501, Shimadzu). Percent cumulative release at each time point was normalized to the total amount of DNA encapsulated in the PLGA microspheres. To confirm if DNA samples in the supernatant contained human BMP-2 cDNA, regular polymerase chain reaction (PCR) was performed with primers 5'-TCTATCCCCACGGAGGAGTTTATC-3' and 5'-GATAGCACTGAGTTCTGTCGGGAC-3', which amplified 695 base pairs fragment from 1547 base pairs BMP-2 cDNA. The PCR products were checked by electrophoresis using $1 \%$ agarose gel. All samples were run in triplicate and data points are shown as means \pm standard deviation.

\section{Cell cultures}

MC3T3-E1 pre-osteoblasts were from mice and purchased from the Chinese Academy of Sciences Cell Bank (Shanghai, People's Republic of China). The cells were cultured in alpha-Minimum Essential Medium ( $\alpha$-MEM) containing $10 \%$ fetal bovine serum (Gibco, Grand Island, NY, USA), $100 \mathrm{U} / \mathrm{mL}$ penicillin, and $100 \mathrm{mg} / \mathrm{mL}$ streptomycin (Gibco) at $37^{\circ} \mathrm{C}$ in a humidified atmosphere of $5 \% \mathrm{CO}_{2}$. The medium was changed every 2-3 days. When cells co-cultured with PLGA@pBMP-2/PEI or PLGA@PEI, the PLGA@pBMP-2/ PEI or PLGA@PEI was recovered by centrifugation and added back to the original culture during medium change. To 
induce osteogenesis, the growth medium was replaced with osteogenic Dulbecco's Modified Eagle's Medium supplemented with $10 \%$ fetal bovine serum, $10 \mathrm{mM} / \mathrm{L}$ sodium $\beta$-glycerol phosphate, L-ascorbic acid (50 mg/L), $10^{-8} \mathrm{M} / \mathrm{L}$ dexamethasone.

\section{Evaluation of transfection efficiency of PLGA@pBMP-2/PEI/FITC in vitro}

FITC was dispersed in sterile water as saturated solution at room temperature. Then, the pBMP-2/PEI nanoparticles at $\mathrm{N} / \mathrm{P}=8$ were prepared. $\mathrm{pBMP}-2 / \mathrm{PEI} / \mathrm{FITC}$ was formed by slowly adding $0.05 \mathrm{~mL}$ FITC solution into $0.55 \mathrm{~mL}$ pBMP-2/PEI solution. The FITC binds to pBMP-2/PEI by hydrogen bonds. Using the W/O/W method described above, the PLGA microspheres, PLGA@pBMP-2/PEI/FITC, were created. MC3T3-E1 cells were seeded at $1.5 \times 10^{5}$ cells/well in 6-well plates. After 24 hours, 0.2 mg of PLGA@pBMP-2/ PEI/FITC was added into the culture. On 1, 3, and 7 days post-transfection, growth medium was replaced with PBS and observed by fluorescence microscopy. In the control group, the same amount of PLGA@PEI microspheres were added into the culture.

\section{RNA extraction and quantitative real-time PCR ( $q P C R$ )}

MC3T3-E1 cells were seeded at $1.5 \times 10^{5}$ cells/well in 6 well plates and cultured in osteogenic medium with PLGA@ pBMP-2/PEI or PLGA@PEI or naïve control. Cells were harvested at 1, 3, 7, and 14 days post-culture. Total RNA was extracted using Qiagen RNeasy mini purification kit (Qiagen, Valencia, CA, USA). RNA was quantitated using a NanoDrop2000 spectrophotometer (Thermo Scientific, West Palm Beach, FL, USA). The cDNAs were synthesized using iScript ${ }^{\mathrm{TM}}$ cDNA synthesis kit (Takara Bio, Tokyo, Japan). The qPCRs were performed using SYBR-Green premix Ex Taq (Takara Bio, Tokyo, Japan) $(\mathrm{n}=3)$ and MxPro Mx3005P real-time PCR detection system (Agilent Technologies, Santa Clara CA, USA). $\beta$-actin was used as a normalization control. Primers used for qPCR are as follows: $\beta$-actin, 5'-CATCCGTAAAGACCTCTATGCCAAC-3'/5'A T G G A G C C A C C G A T C C A C A - $3^{\prime} ; A L P$, 5'-CTCAACACCAATGTAGCCAAGAATG-3' $/ 5^{\prime}$-GGCAG CGGTTACTGTGGAGA-3'; RUNX2, 5'-GCACAAACATG GCCAGATTCA-3'/5'-AAGCCATGGTGCCCGTTAG-3'; COLL $I, 5^{\prime}$-GACATGTTCAGCTTTGTGGACCTC-3' $/ 5^{\prime}$ G G G A C C C T TA G G C C A T T G T G TA - 3' ; $S P 7$, 5'-AAGTTATGATGACGGGTCAGGTACA-3'/5' AGAAATCTACGAGCAAGGTCTCCAC-3'.
Primers were synthesized commercially (TaKaRa Biotechnology [Dalian] Co, Ltd, Dalian, People's Republic of China).

Human BMP-2 enzyme-linked immunosorbent assay (ELISA) and alizarin red staining

MC3T3-E1 cells were seeded at $1.5 \times 10^{5}$ cells/well in 6-well plates and cultured in osteogenic medium with PLGA@ pBMP-2/PEI or PLGA@PEI. Cells cultured without any PLGA microspheres were naïve controls. Cell culture medium was collected on days 1, 3, 7, and 14 for ELISA analysis. Human BMP-2 concentration was determined with a BMP-2 ELISA kit (BOSTER, Wuhan, People's Republic of China). When the culture medium was collected, microspheres were recovered by centrifugation, resuspended in fresh growth medium, and added into the corresponding culture again. After the culture medium was collected on day 14, cells were stained by Alizarin red to assess mineralization. For Alizarin red staining, the cells were fixed in $95 \%$ ethanol at room temperature for 10 minutes and stained with $0.1 \%$ solution of Alizarin red at $\mathrm{pH} 4.1-4.5$ for 30 minutes.

\section{In vivo implantation}

The animal protocol used for this study was approved by the Institutional Animal Care and Use Committee of Jilin University. Twenty-four male Wistar rats (4 weeks old, body weight 140-160 g) were used in this study. Surgery was done under anesthetic ( $60 \mathrm{mg} / \mathrm{kg}$ of ketamine and $8 \mathrm{mg} / \mathrm{kg}$ of xylazine). The surgical site was shaved and scrubbed with iodine. For the calvarial defect model, an incision was made in a sagittal plane across the cranium using a $5 \mathrm{~mm}$ diameter hollow trephine bur (3i Implant Innovation, Palm Beach Gardens, FL, USA); a full thickness flap was dissected to expose calvarial bone and was copiously irrigated with saline. After the removal of the trephined calvarial disk, a gelatin sponge with PLGA loaded with PLGA@PEI or PLGA@pBMP-2/PEI was applied to the defect site. Rats with no defect treatment were used as negative controls (Figure 4D). Each group consisted of four rats. Finally, 4.0 (1.5 metric) nylon sutures were used to close the pericardium and overlying skin.

\section{Analysis of in vivo experimental samples}

At 4 and 8 weeks post-surgery bone formation was evaluated under anesthetic using micro-computed tomography (CT) (INVEON PET/CT system, Siemens Healthcare Global, Siemens AG, Erlangen, Germany). Then, rats were perfused with $10 \%$ neutral buffered formalin, and bone samples were decalcified in ethylene diamine tetraacetic acid for 1 month and embedded in 
paraffin. Serial sections $4 \mu \mathrm{m}$ thick were cut through the center of circular calvarial defects. Sections were stained with hematoxylin and eosin (H\&E) and immunohistochemistry was performed. For immunohistochemistry staining, endogenous peroxidase in the tissue section was blocked with $1 \%$ hydrogen peroxide for 10 minutes then further blocked with $1 \%$ goat serum in PBS for 30 minutes. The primary antibody used was polyclonal rabbit anti-human BMP-2 antibody (Wuhan Boster Biological Technology Ltd, Wuhan, People's Republic of China). An immunohistochemistry staining kit (Vector Laboratories, Burlingame, CA, USA) was used for further staining. The negative control slide was not incubated with polyclonal rabbit anti-human BMP-2 antibody, but instead with rabbit immunoglobulin (Ig)G. Finally, sections were counterstained with hematoxylin and mounted.

\section{Statistical analyses}

Results are expressed as the mean \pm standard deviation (SD). One-way analysis of variance, followed by a Tukey's test, were employed as appropriate to determine the statistical significance observed. Statistical significance was accepted when $P<0.05$.

\section{Results}

\section{Characteristics of $\mathrm{PBMP}-2 / \mathrm{PEI}$} nanoparticles and PLGA microspheres

Data showed that diameter of pBMP-2/PEI nanoparticles was $317.4 \pm 21 \mathrm{~nm}$ (Figure 2A) and zeta potential of pBMP-2/PEI nanoparticles was $14.2 \pm 2.1 \mathrm{mV}$ (Figure 2B). Data from SEM showed that PLGA@pBMP-2/PEI exhibited spherical particles with smooth and dense surfaces, and with good dispersion (Figure 2C). The diameter of these microspheres was 3-15 $\mu \mathrm{m}$ (Figure 2C). Figure 2E is the in vitro cumulative release profile of pBMP-2 plasmid DNA; the supernatant was analyzed using a ultraviolet (UV) spectrophotometer. This figure shows that the pBMP-2 released from the PLGA microspheres has two phases: (1) an exponential release phase from day 1 to day 30 ;
A

$\frac{D}{0}$
$\frac{0}{0}$
$\stackrel{ \pm}{=}$

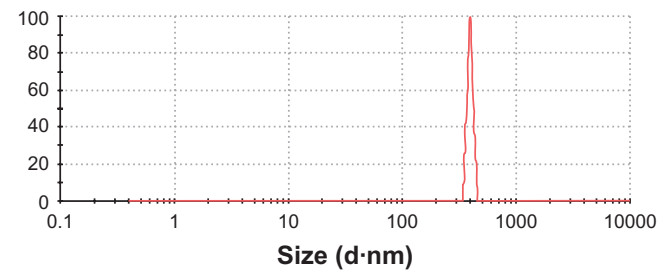

B

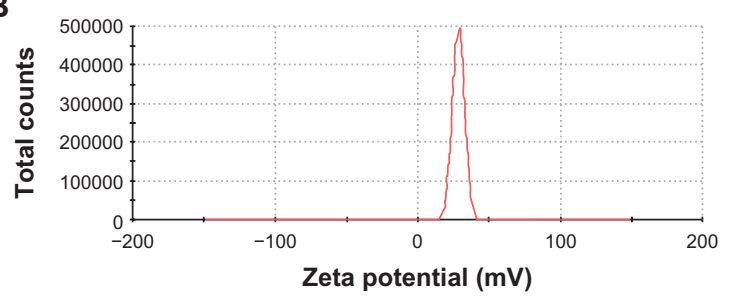

E

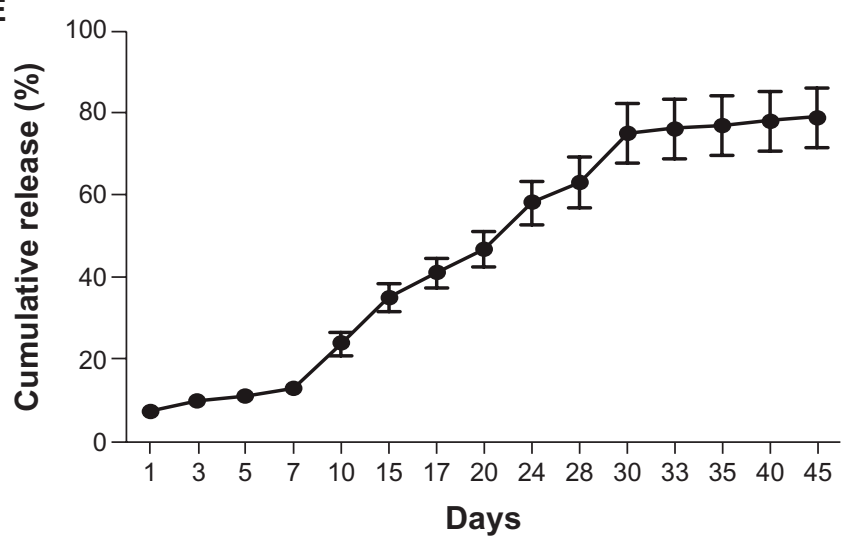

C

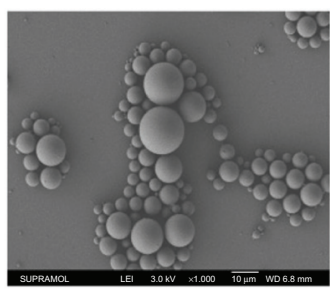

D

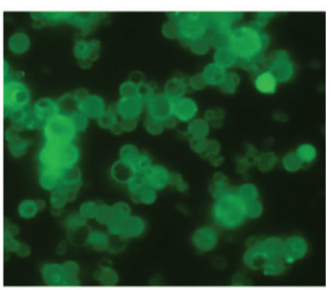

$\mathbf{F}$

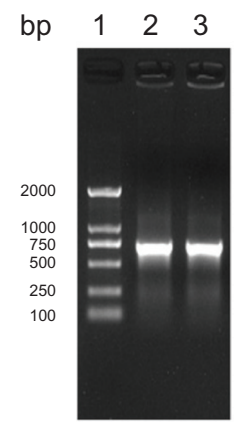

Figure 2 Physical characteristics of pBMP-2/PEI nanoparticles, morphology of PLGA@pBMP-2/PEI, and release assay of pBMP-2. (A) Size distribution of BMP-2/PEI nanoparticles; (B) Zeta potential of BMP-2/PEI nanoparticles; (C) Scanning electron microscope image of PLGA@PBMP-2/PEl; (D) Fluorescence image of PLGA@pBMP-2/ PEI/FITC; (E) pBMP-2 release profile from PLGA@PBMP-2/PEI in PBS; (F) PCR assays to confirm pBMP-2 releasing into the PBS buffer. Lane I: DNA marker. Lane 2: PCR amplicon of original pBMP-2 plasmid. Lane 3: PCR amplicon from day 7 PBS buffer

Abbreviations: PLGA@pBMP-2/PEI/FITC, poly(lactic-co-glycolic acid)@plasmid of bone morphogenetic protein 2/polyethlenimine/fluorescein isothiocyanate; bp, base pair; PCR, polymerase chain reaction; PBS, phosphate buffered salin. 
(2) a plateau phase from day 31 to day 35. After 35 days, no further release was found, and data from the UV spectrophotometer showed that there was 19\% of pBMP-2 in the PLGA@ pBMP-2/PEI, which indicated that $~ 19 \%$ of pBMP-2 plasmid DNA was difficult to fully release from the PLGA microspheres in vitro (Figure $2 \mathrm{E}$ ). Figure $2 \mathrm{~F}$ demonstrated that 695 base pairs of BMP-2 cDNA could be amplified by PCR assay from day 7 supernatant. This finding further indicated that the optical density (OD) values, which were measured by UV spectrophotometer for the release profile, were pBMP-2 plasmid released from PLGA microspheres. In this study, the entrapment efficiency of PLGA microspheres was $40.3 \% \pm 7.3 \%$ which was measured by spectrophotofluorometer. Drug-loading rate of PLGA@pBMP-2/PEI was 1.7\% $0.5 \%$ (w/w), which was found by calculating the percentage of gene encapsulated in PLGA microspheres from the total amount of microspheres.

\section{Characteristics of PLGA@/pBMP-2/PEI transfection in MC3T3-EI cells in vitro}

To evaluate the ability of PLGA@/pBMP-2/PEI to transfect MC3T3-E1 cells, the PLGA@/pBMP-2/PEI microspheres were labeled with FITC (Figure 2D). Figure 2D indicates that the FITC evenly labeled the PLGA@/pBMP-2/PEI. AfterPLGA@/pBMP-2/PEI/FITC were co-cultured with the MC3T3-E1 cells, Figure 3A and B show that pBMP-2/PEI/ FITC, which was released from PLGA@/pBMP-2/PEI/FITC, transfected $\sim 8 \%$ of MC3T3-E1 cells on day 3 and $>50 \%$ of MC3T3-E1 cells on day 7. These results indicate that the pBMP-2/PEI/FITC complex was gradually released from PLGA and efficiently transfected MC3T3-E1 cells in vitro.

To confirm if there were pBMP-2 plasmids in the transfected cells, DNA samples were extracted from the transfected cells and PCR was performed to amplify part of human BMP-2 cDNA. Results from Figure 3C demonstrated that, as expected, human BMP-2 PCR product could only be amplified from the transfected MC3T3-E1 cells with the PLGA@/ pBMP-2/PEI/FITC. This finding suggests that the plasmid pBMP-2 was present in the transfected cells. Next, it was important to know if the transfected cells could secrete human BMP-2 protein. Data from Figure 3D clearly show that the MC3T3-E1 cells transfected with the PLGA@/pBMP-2/PEI could secrete human BMP-2 protein on days 7 and 14.

\section{Effects of PLGA@pBMP-2/PEI on osteogenic differentiation of MC3T3-EI cells}

To determine whether $\mathrm{pBMP}-2 / \mathrm{PEI}$ can induce osteogenic differentiation of MC3T3-E1 cells in vitro, gene expressions of $A L P, R U N X 2, S P 7$, and COLL $I$ were measured after MC3T3-E1 cells were transfected with PLGA@pBMP-2/ PEI or PLGA@PEI and cultured in osteogenic conditioned media for 1, 3, 7, and 14 days. Data from Figure 4 show that gene expressions of $A L P, R U N X 2, S P 7$, and COLL I significantly increase after day 3 compared to the PLGA@, PEI and the naïve control group $(P<0.05)$. Interestingly, the peak of gene expression of $A L P, S P 7$, and COLL I was on day 14, while the peak of gene expression of $R U N X 2$ was on day 7 (Figure 4). These data clearly suggest that the PLGA@pBMP-2/PEI could induce differentiation of transfected MC3T3-E1 cells in vitro from pre-osteoblasts to osteoblasts.

Next, Alizarin red staining was used to check whether the PLGA@/pBMP-2/PEI could affect calcium deposition of MC3T3-E1 cells. Figure 3E and F demonstrate that red calcium deposition nodules markedly increased from MC3T3-E1 cells transfected with the PLGA@/pBMP-2/PEI compared to the PLGA@PEI. All of the above data suggest that the PLGA@/pBMP-2/PEI/FITC could release pBMP-2/ $\mathrm{PEI} / \mathrm{FITC}$, transfect target cells, and produce functional therapeutic human BMP-2 protein.

\section{Effects of PLGA@pBMP-2/PEI on bone formation in an in vivo model}

In vivo experiments were performed using gelatin sponges loaded with or without PLGA@pBMP-2/PEI implanted into cranial bone defects, and bone formation was observed with micro-CT and H\&E staining after 4 or 8 weeks post-surgery (Figures 5-7). The PLGA@pBMP-2/PEI microspheres were found in the gelatin sponge (Figure 5B). At 8 weeks, new bone in the defect area was observed only in the PLGA@ pBMP-2/PEI group (Figure 6). Quantitative analysis demonstrated that the residual defect volume of the PLGA@, pBMP-2/PEI group (16.68 $\left.\pm 1.7 \mathrm{~mm}^{3}\right)$ was significantly lower than that of the PLGA@PEI group (19.14 $\left.\pm 2.8 \mathrm{~mm}^{3}\right)$ or the non-implant group $\left(21.4 \pm 3.02 \mathrm{~mm}^{3}\right)(P<0.05)$, while there was no difference between the PLGA@PEI group and the non-implant group $(P>0.05)$. Further histological analyses confirmed that new bone formed not only along the periphery of the defect area but also in the center of defect area in the PLGA@pBMP-2/PEI group at both 4 weeks and 8 weeks (Figure 7E-J), especially at 8 weeks. The new bone formation, however, was observed only at the margin of the defect area in the PLGA@PEI group and the non-implant group at both 4 weeks and 8 weeks (Figure 7A-D). In the center of the defect area, only connective tissues were observed in the PLGA@PEI 
A

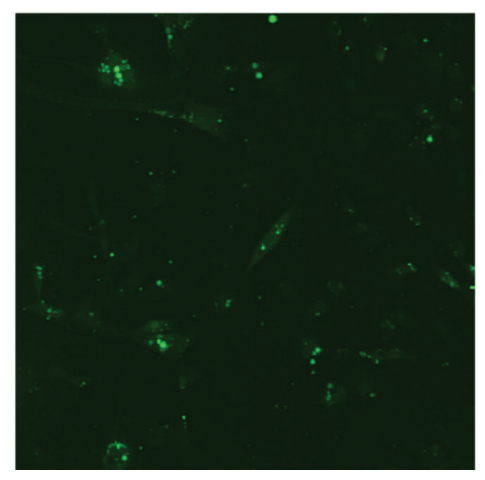

B

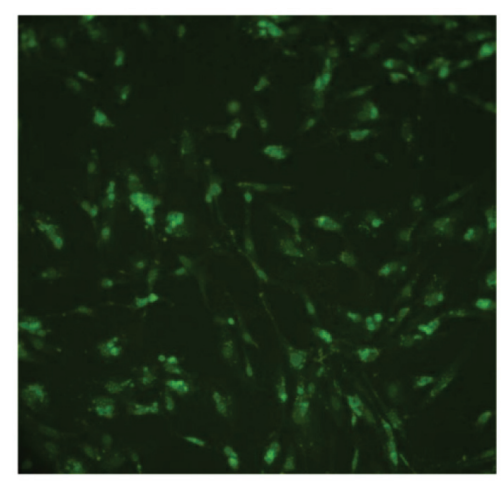

C

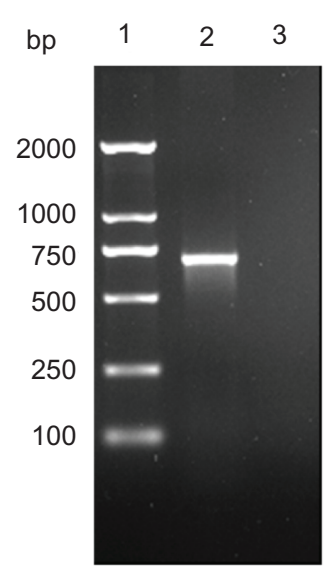

E

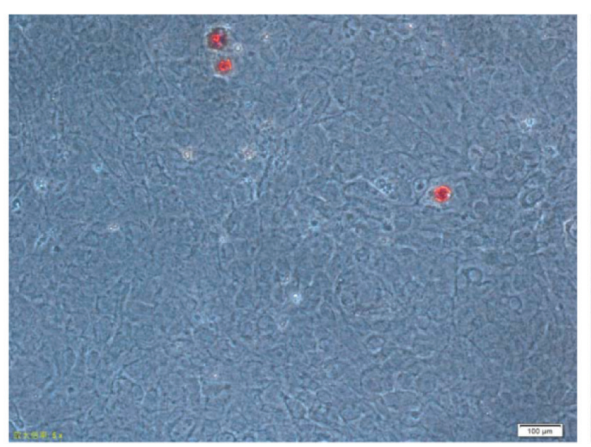

D

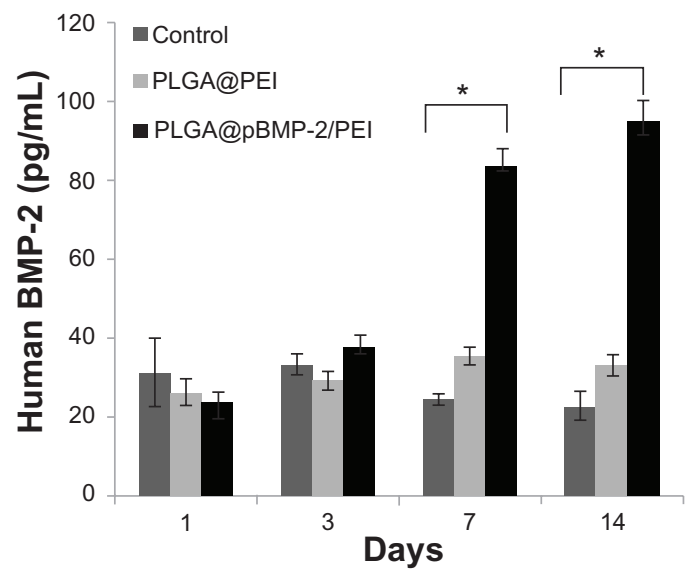

$\mathbf{F}$

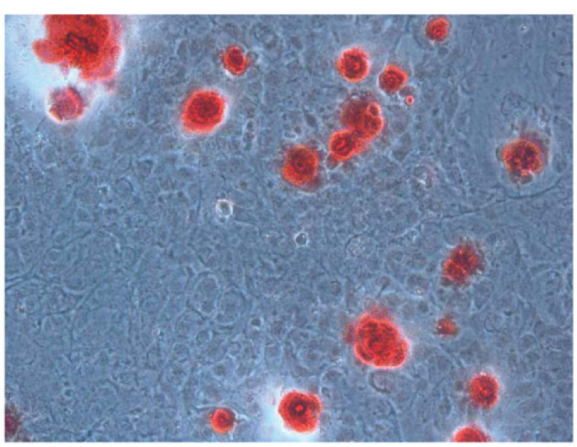

Figure 3 Evaluate properties of PLGA@pBMP-2/PEl in vitro. (A) Transfection ability of PLGA@PBMP-2/PEI/FITC in MC3T3-EI cells at day 3; (B) Transfection ability of PLGA@ PBMP-2/PEI/FITC in MC3T3 cells at day 7; (C) PCR assays to confirm pBMP-2 in MC3T3 cells. Lane I: DNA marker; Lane 2: PCR amplicon from PLGA@PBMP-2/PEI transfected MC3T3 cells; Lane 3: PCR from MC3T3 cells; (D) Human BMP-2 ELISA assays to confirm human BMP-2 secretion from transfected MC3T3 cells. (E) Calcium deposition analysis of PLGA@PEl group on day 14 using Alizarin red staining; (F) Calcium deposition analysis of PLGA@pBMP-2/PEl group on day I4 using Alizarin red staining.

Note: *significant difference $(p<0.0 \mathrm{I})$.

Abbreviations: PLGA@PEI, poly(lactic-co-glycolic acid)@polyethlenimine; PLGA@pBMP-2/PEI, poly(lactic-co-glycolic acid)@plasmid of bone morphogenetic protein 2/ polyethlenimine; PLGA@PBMP-2/PEI/FITC, poly(lactic-co-glycolic acid)@plasmid of bone morphogenetic protein 2/polyethlenimine/fluorescein isothiocyanate; bp, base pair; PCR, polymerase chain reaction; ELISA, enzyme-linked immunosorbent assay.

group and non-implant group at both 4 weeks and 8 weeks (Figure 7A-D). Osteoid and osteoblasts were found around the new bone (Figure $7 \mathrm{G}-\mathrm{J}$ ). In the original defect margin, reactive new bones in the PLGA@pBMP-2/PEI group were much more than that of the PLGA@PEI group and the nonimplant group (Figure 7I and J).
Immunohistochemistry staining demonstrated that human BMP-2 was located in the cytoplasm of mesenchymal cells adjacent to the new bone area in the PLGA@pBMP-2/PEI group at 8 weeks while the two control groups were negative (Figure $7 \mathrm{~K}$ and L). The above in vivo data suggest that human BMP-2 significantly 

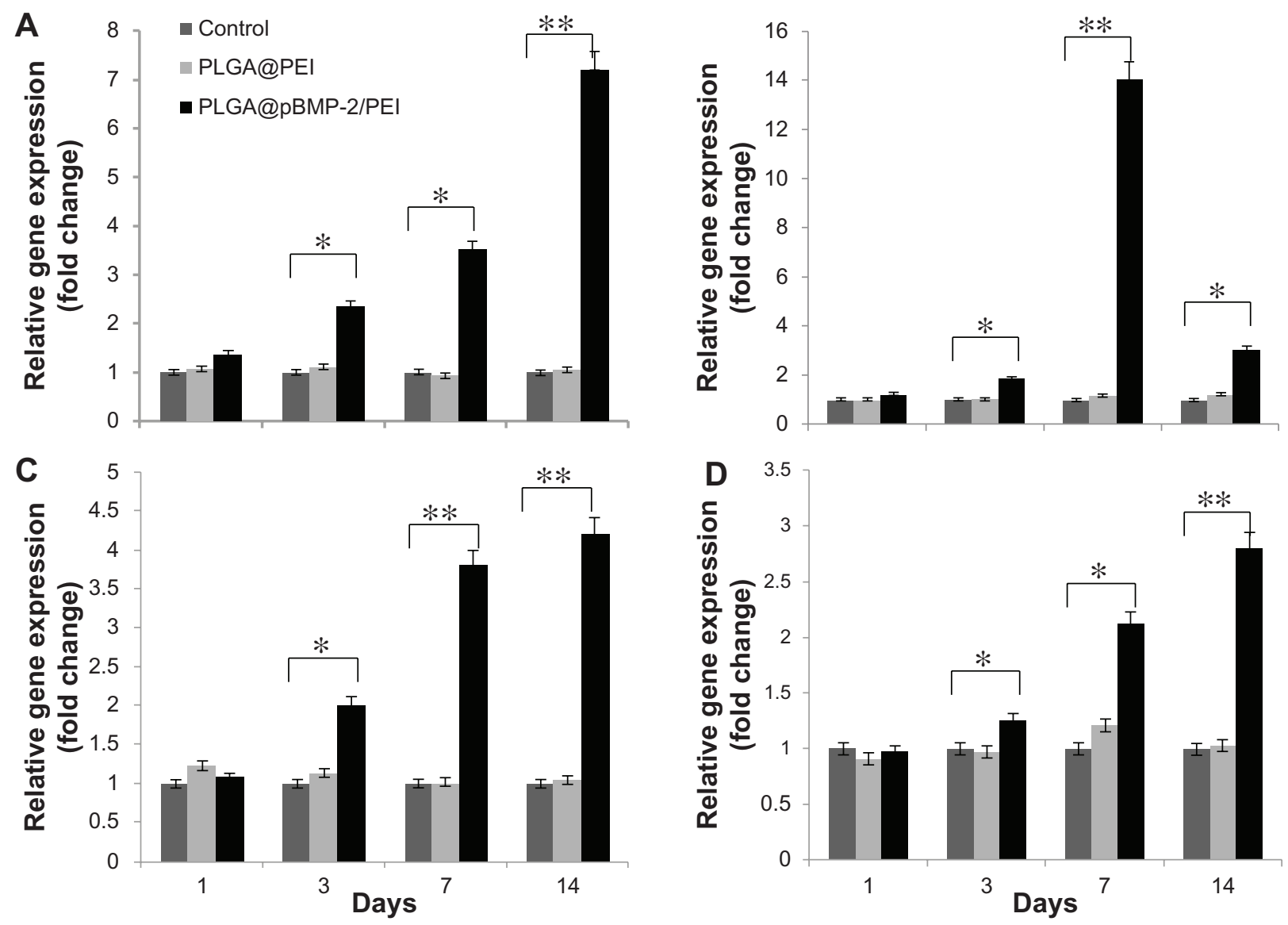

Figure 4 Cell differentiation analysis of MC3T3-EI cells after I, 3, 7, and I4 days post-transfection using qPCR assays. (A) Alkaline phosphatase gene expression; (B) runt-related transcription factor 2 gene expression; (C) SP7 gene expression; (D) I type collagen gene expression. All assays were repeated three times. Data shown are mean values \pm SD. Notes: $* P<0.05 ; * * P<0.01$.

Abbreviations: PLGA@PEI, poly(lactic-co-glycolic acid)@polyethlenimine; PLGA@pBMP-2/PEl, poly(lactic-co-glycolic acid)@plasmid of bone morphogenetic protein 2/ polyethlenimine; qPCR, Real-Time quantitative polymerase chain reaction; SD, standard deviation.
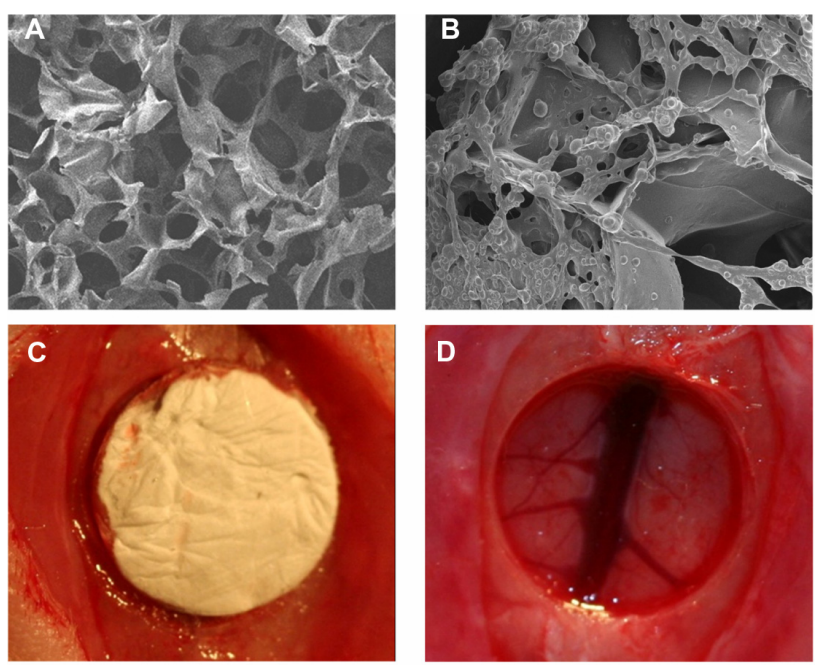

Figure 5 SEM image of gelatin sponge with or without PLGA@pBMP-2/PEI and representative photos of bone defect with or without treatment. (A) SEM image of gelatin sponge without PLGA@pBMP-2/PEl; (B) SEM image of gelatin sponge with PLGA@PBMP-2/PEl; (C) Cranial bone defect with gelatin sponge loaded PLGA@pBMP-2/PEl treatment; (D) Cranial bone defect without treatment (5 mm diameter).

Abbreviations: SEM, Scanning Electron Microscopy; PLGA@pBMP-2/PEI, poly(lacticco-glycolic acid)@plasmid of bone morphogenetic protein 2/polyethlenimine. stimulated the new bone formation in the bone defect area.

\section{Discussion}

In order to establish an efficient means of repairing bone defects, we created pBMP2/PEI nanoparticles, which to obtain efficient transfection were dependent on the positively charged PEI linked to the negatively charged

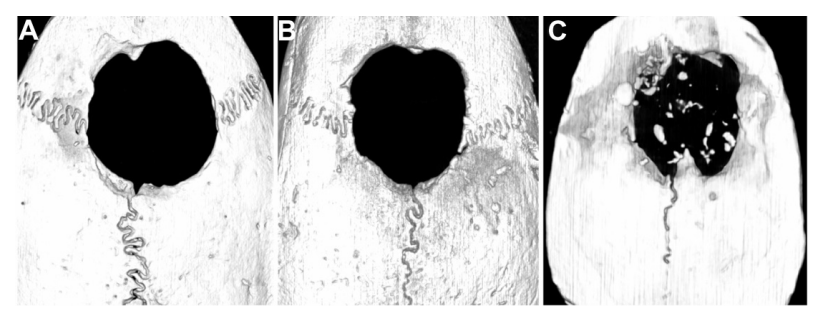

Figure 6 Micro-CT images of rat cranial bone after 8 weeks post-implantation. (A) Negative control; (B) Gelatin sponge loaded with PLGA@PEI; (C) Gelatin sponge loaded with PLGA@pBMP-2/PEI.

Abbreviation: PLGA@PEl, poly(lactic-co-glycolic acid)@polyethlenimine; PLGA@ pBMP-2/PEl, poly(lactic-co-glycolic acid)@plasmid of bone morphogenetic protein 2/ polyethlenimine; Micro-CT, micro-computed tomography. 


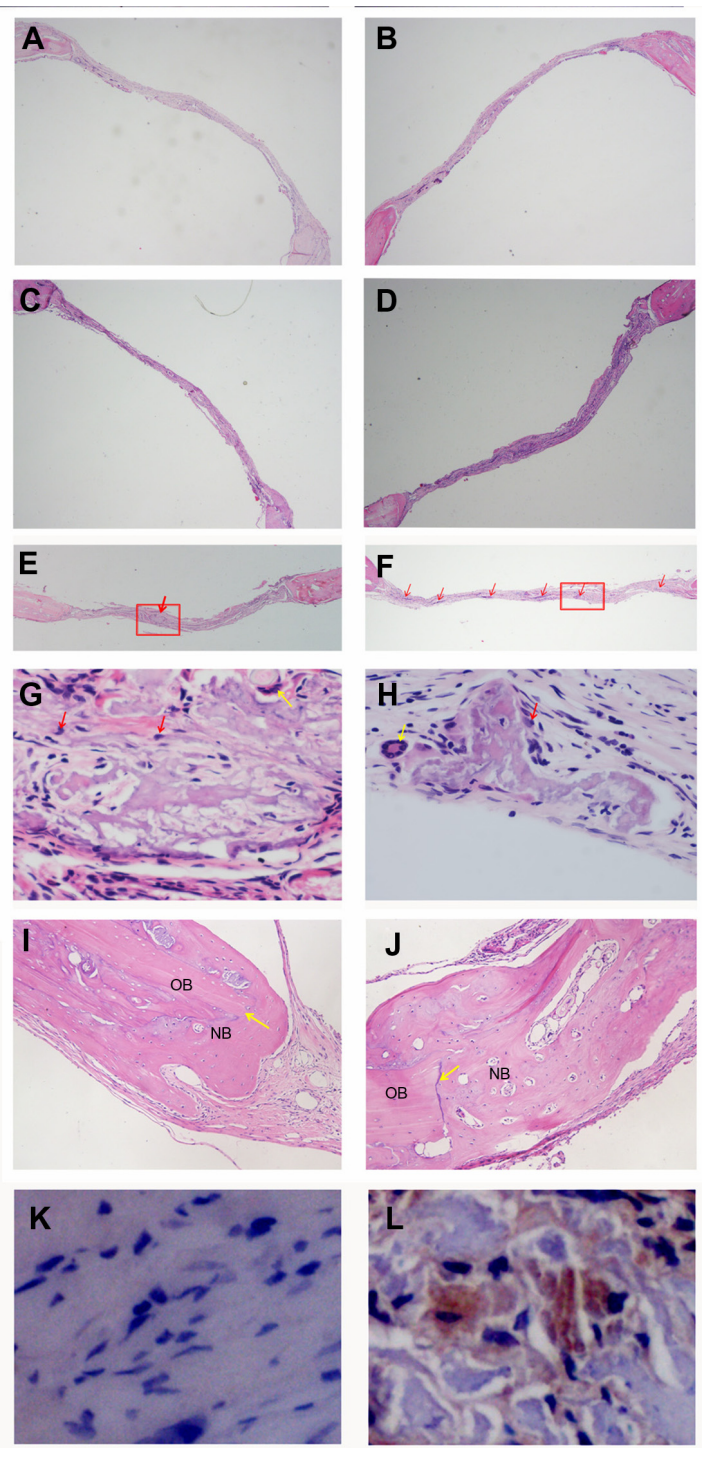

Figure $7 \mathrm{H} \& \mathrm{E}$ staining and immunohistochemistry staining after 4 or 8 weeks postimplantation. (A) H\&E staining of non-implant control after 4 weeks $(\times 40)$; (B) H\&E staining of non-implant control after 8 weeks $(\times 40)$; (C) H\&E staining of PLGA@ PEl group after 4 weeks $(\times 40)$; (D) H\&E staining of PLGA@PEI group after 8 weeks ( $\times 40)$; (E) H\&E staining of PLGA@PBMP-2/PEI group after 4 weeks $(\times 40)$, red arrow indicates new bone; (F) H\&E staining of PLGA/pBMP-2/PEI group after 8 weeks $(\times 40)$, red arrows indicate new bone; $(\mathbf{G})$ Enlarged image of new bone area from Figure 6E. Red arrows indicate osteoblasts and yellow arrow indicates a macrophages phagocytized foreign materials; $(\mathbf{H})$ Enlarged image of new bone area from Figure 6F. Red arrow indicates osteoblasts, and yellow arrow indicates a macrophages phagocytized foreign materials; (I) H\&E staining of PLGA@pBMP-2/PEI group after 4 weeks $(\times 200)$. OB: original bone (or lamellar bone); NB: new bone (or woven bone); Yellow arrow indicates the edge of the defect area; (J) H\&E staining of PLGA@pBMP$2 / \mathrm{PEI}$ group after 8 weeks $(\times 200)$. Yellow arrow indicates the edge of the defect area; (K) Human BMP-2 immunohistochemistry staining of PLGA@PEl group; (L) Human BMP-2 immunohistochemistry staining of PLGA/PBMP-2/PEI group.

Abbreviations: H\&E, hematoxylin and eosin stain; PLGA@PEl, poly(lactic-co-glycolic acid)@polyethlenimine; PLGA@pBMP-2/PEl, poly(lactic-co-glycolic acid)@plasmid of bone morphogenetic protein 2/polyethlenimine;

pBMP-2 plasmid, and the weak negatively charged cell membrane. Then, using the slow release property of PLGA, we developed novel PLGA microspheres-PLGA@ pBMP2/PEI. Data demonstrated that the human BMP-2
plasmid/PEI could be efficiently released from the PLGA microsphere, transfect target cells in vitro and in vivo, and stimulate cell differentiation in vitro and bone formation in vivo.

In vivo bone formation requires a relatively long time period. To meet this biological requirement, a sustained gene expression of human BMP-2 or slow release of plasmid BMP-2, with the ability to transfect local target cells at the bone defect area was required. It is well-known that BMP-2 protein is rapidly degraded in vivo. ${ }^{23}$ Previous studies showed that the PLGA possesses the ability to slowly release loaded particles. ${ }^{24-27}$ Therefore, PLGA was used to create PLGA@, pBMP-2/PEI microspheres and gradually release pBMP-2/ PEI nanoparticles for a relatively longer period of time.

We demonstrated that the pBMP-2/PEI nanoparticles could successfully be encapsulated into PLGA microspheres and be slowly released from PLGA (Figure 2). In this study, PLGA entrapment efficiency was about $40.3 \% \pm 7.3 \%$, and drug loading rate was $1.7 \% \pm 0.5 \%(\mathrm{w} / \mathrm{w})$. While in our study the encapsulation efficiency could be over $50 \%$ if the concentration of PLGA (50:50) was $4 \%(\mathrm{w} / \mathrm{v})$, previous studies showed that increasing PLGA concentration can increase the encapsulation efficiency. ${ }^{28}$ Increasing the concentration of PLGA, however, would reduce the drug loading rate and prolong the degradation time of the polymer and the release rate of pBMP-2, which affected bone defect repair in our study.

Conventional microparticles can result in $>50 \%$ burst release within the first day. ${ }^{29}$ One study has shown that sustained release of growth factor efficiently enhances healing compared to burst release. ${ }^{30-32}$ Sustained release, rather than burst release, of recombinant human BMP-2 from polymer scaffolds promoted more bone formation in both orthotopic ${ }^{31}$ and ectopic models. ${ }^{32}$ It is known that microsphere size significantly affects in vitro release kinetics. ${ }^{33}$ In the present study, the size of PLGA@pBMP-2/PEI microspheres was 3-15 $\mu \mathrm{m}$ (Figure 2C). The pBMP-2/PEI nanoparticles in the smaller microspheres were released easily and fast because the pBMP-2/PEI nanoparticles were distributed near the surface while the pBMP-2/PEI nanoparticles released in the bigger microspheres had more difficultly and less speed because the pBMP-2/PEI could also locate inside the microspheres. Different sizes of PLGA microspheres created in this study have the potential to enhance persistent release of the pBMP-2/PEI nanoparticles from PLGA microspheres. The release profile of PLGA@pBMP-2/PEI in this study showed stable release until 35 days without a burst release. The initial release rate is lower because the pBMP-2/PEI nanoparticles on the surface of PLGA microspheres could 
be removed during washing with distilled water. Following a short, slow release at 24 hours was an exponential release phase of pBMP-2/PEI nanoparticles because of the PLGA polymer erosion and degradation.

Our data also demonstrated that the pBMP-2/PEI efficiently transfect MC3T3-E1 cells (Figure 3). Importantly, the transfected cells, MC3T3-E1 cells, could produce human BMP-2 protein and increase gene expressions of $A L P, R U N X 2, S P 7$, and COLL I to induce differentiation of pro-osteoblast cells (Figures 3 and 4). These results indicate that the PEI could condense DNA and protect DNA from being damaged during primary emulsion. After a large amount of foam formed when the pBMP-2/PEI solution was homogenized with PVA during the second emulsion, it created a membrane layer that further prevented the plasmid DNA damage.

It is well-known that BMP-2 is able to induce osteogenic differentiation of stem cells in vitro, ${ }^{34,35}$ and to enhance bone formation in vivo. ${ }^{36}$ In our study, BMP-2 cDNA delivered using PLGA microspheres has demonstrated that it can be secreted from transfected MC3T3-E1 cells, stimulate its differentiation, matrix mineralization, and bone formation. Interestingly and importantly, in our study we also observed human BMP-2 expression in the mesenchymal cells adjacent to the new bone in vivo at 8 weeks post-implantation with a gelatin sponge impregnated with PLGA@pBMP-2/ PEI (Figures 6 and 7). This finding indicates that pBMP-2/ PEI transfected local stem cells/osteoblast cells resulting in significantly increased new bone formation at 4 weeks postimplantation with the PLGA@pBMP-2/PEI, especially at 8 weeks (Figures 6 and 7). The study also showed that pBMP-2/ PEI was slowly released from the PLGA@pBMP-2/PEI; other research has shown that slow release is more effective in bone formation than burst release. ${ }^{37}$

\section{Conclusion}

Our study demonstrated that our PLGA-nanoparticle delivery system efficiently and functionally delivered BMP-2 cDNA in vitro and in vivo, resulting in differentiation of osteoblasts and new bone formation in the bone defect area. These findings indicate that our delivery system has a potential clinical application, although we still need to optimize this system further.

\section{Acknowledgments}

This work was supported by the National Science Foundation of China (Grant No 30830108, 81271111), Doctoral Fund of Ministry of Education of China (Grant No 233200801830063),
Science and Technology Development projects of Jilin Province (No 201101051), Jilin Provincial Health Department research projects (No 2012S017), and Fundamental Research Project of the Central Universities, People's Republic of China.

\section{Disclosure}

The authors report no other conflicts of interest in this work.

\section{References}

1. Meyer U, Joos U, Wiesmann HP. Biological and biophysical principles in extracorporeal bone tissue engineering: part I. Int J Oral Maxillofac Surg. 2004;33:325-332.

2. Hogan BL. Bone morphogenetic proteins in development. Curr Opin Genet Dev. 1996;6:432-438.

3. Kang SW, Kim JS, Park KS, et al. Surface modification with fibrin/ hyaluronic acid hydrogel on solid-free form-based scaffolds followed by BMP-2 loading to enhance bone regeneration. Bone. 2011;48:298-306.

4. Boerckel JD, Kolambkar YM, Dupont KM, et al. Effects of protein dose and delivery system on BMP-mediated bone regeneration. Biomaterials. 2011;32:5241-5251.

5. Kirby GTS, White LJ, Rahman, et al. PLGA-Based microparticles for the sustained release of BMP-2. Polymers. 2011;3:571-586.

6. Ji Y, Xu GP, Zhang ZP, Xia JJ, Yan JL, Pan SH. BMP-2/PLGA delayedrelease microspheres composite graft,sel ection of bone particulate diameters, and prevention of aseptic inflammation for bone tissue engineering. Ann Biomed Eng. 2010;38:632-639.

7. Park J, Lutz R, Felszeghy E, et al. The effect on bone regeneration of a liposomal vector to deliver BMP-2 gene to bone grafts in peri-implant bone defects. Biomaterials. 2007;28:2772-2782.

8. Riew KD, Wright NM, Cheng S, Avioli LV, Lou J. Induction of bone formation using a recombinant adenoviral vector carrying the human BMP-2 gene in a rabbit spinal fusion model. Calcif Tissue Int. $1998 ; 63: 357-360$

9. Gelse K, von der MK, Aigner T, Park J, Schneider H. Articular cartilage repair by gene therapy using growth factor-producing mesenchymal cells. Arthritis Rheum. 2003;48:430-441.

10. Wegman F, Bijenhof A, Schuijff L, Oner FC, Dhert WJ, Alblas J. Osteogenic differentiation as a result of BMP-2 plasmid DNA based gene therapy in vitro and in vivo. Eur Cell Mater. 2011;21;230-242.

11. Nie H, Ho ML, Wang CK, Wang, CH, Yin-Chih F. BMP-2 plasmid loaded PLGA/HAp composite scaffolds for treatment of bone defects in nude mice. Biomaterials. 2009;30:892-901.

12. Chumakova OV, Liopo AV, Andreev VG, et al. Composition of PLGA and PEI/DNA nanoparticles improves ultrasound-mediated gene delivery in solid tumors in vivo. Cancer Lett. 2008;261:215-225.

13. Nguyen HK, Lemieux P, Vinogradov SV. Evaluation of polyetherpolyethyleneimine graft copolymers as gene transfer agents. Gene Ther. 2000;7:126-138.

14. Godbey WT, Wu KK, Mikos AG. Poly(ethylenimine) and its role in gene delivery. J Control Release. 1999;60:149-160.

15. Zhang S, Xu Y, Wang B, Qiao W, Liu D, Li Z. Cationic compounds used in lipoplexes and polyplexes for gene delivery. J Control Release. 2004;100:165-180.

16. Kempen $\mathrm{DH}, \mathrm{Lu} \mathrm{L}, \mathrm{Hefferan} \mathrm{TE}$, et al. Retention of in vitro and in vivo BMP-2 bioactivities in sustained delivery vehicles for bone tissue engineering. Biomaterials. 2008;29:3245-3252.

17. Ruhe PQ, Boerman OC, Russel FG, Mikos AG, Spauwen PH, Jansen JA. In vivo release of rhBMP-2 loaded porous calcium phosphate cement pretreated with albumin. J Mater Sci Mater Med. 2006;17:919-927.

18. Wozney JM. Overview of bone morphogenetic proteins. Spine. 2002;27:S2-S8.

19. Seeherman H, Wozney J, Li R. Bone morphogenetic protein delivery systems. Spine. 2002;27:S16-S23. 
20. Uludag H, D’Augusta D, Palmer R, Timony G, Wozney J. Characterization of rhBMP-2 pharmacokinetics implanted with biomaterial carriers in the rat ectopic model. J Biomed Mater Res. 1999;46: 93-202.

21. Ho J, Wang H, Forde GM. Process considerations related to the microencapsulation of plasmid DNA via ultrasonic atomization. Biotechnol Bioeng. 2008;101:172-181.

22. Andreas K, Zehbe R, Kazubek M, et al. Biodegradable insulinloaded PLGA microspheres fabricated by three different emulsification techniques: Investigation for cartilage tissue engineering. Acta Biomater. 2011;7:1485-1495.

23. Sellers RS, Zhang R, Glasson SS, et al. Repair of articular cartilage defects one year after treatment with recombinant human bone morphogenetic protein-2 (rhBMP-2). J Bone Joint Surg Am. 2000;82:151-160.

24. Jain RA. The manufacturing techniques of various drug loaded biodegradable poly(lactide-co-glycolide) (PLGA) devices. Biomaterials. 2000;21:2475-2490.

25. Nath SD, Son S, Sadiasa A, Min YK, Lee BT. Preparation and characterization of PLGA microspheres by the electrospraying method for delivering simvastatin for bone regeneration. Int J Pharm. 2013;443:87-94.

26. Freiberg S, Zhu XX. Polymer microspheres for controlled drug release. Int J Pharm. 2004;282:1-18.

27. Klose D, Siepmann F, Elkharraz K, Siepmann J. PLGA-based drug delivery systems: importance of the type of drug and device geometry. Int J Pharm. 2008;354:95-103.

28. Fu X, Ping Q, Gao Y. Effects of formulation factors on encapsulation efficiency and release behaviour in vitro of huperzine A-PLGA microspheres. J Microencapsul. 2005;22:705-714.
29. McGinity JW, O’Donnell PB. Preparation of microspheres by the solvent evaporation technique. Adv Drug Deliv Rev. 1997;28:25-42.

30. Jung MR, Shim IK, Chung HJ, et al. Local BMP-7 release from a PLGA scaffolding-matrix for the repair of osteochondral defects in rabbits. J Control Release. 2012;162:485-91.

31. Kim SS, Gwak SJ, Kim BS. Orthotopic bone formation by implantation of apatite-coated poly (lactide-co-glycolide)/hydroxyapatite composite particulates and bone morphogenetic protein-2. J Biomed Mater Res A. 2008;87:245-253.

32. Jeon O, Song SJ, Yang HS, et al. Long-term delivery enhances in vivo osteogenic efficacy of bone morphogenetic protein-2 compared to shortterm delivery. Biochem Biophys Res Commun. 2008;369:774-780.

33. Liu X, Ma PX. The nanofibrous architecture of poly (L-lactic acid)-based functional copolymers. Biomaterials. 2010;31:259-269.

34. Rauch F, Lauzier D, Croteau S, Travers R, Glorieux FH, Hamdy R. Temporal and spatial expression of bone morphogenetic protein-2, -4 , and -7 during distraction osteogenesis in rabbits. Bone. 2000;27:453-459.

35. Katagiri T, Yamaguchi A, Ikeda T, et al. The nonosteogenic mouse pluripotent cell line, C3H10T1/2, is induced to differentiate into osteoblastic cells by recombinant human bone morphogenetic protein-2. Biochem Biophys Res Commun. 1990;172:295-299.

36. Puleo DA. Dependence of mesenchymal cell responses on duration of exposure to bone morphogenetic protein-2 in vitro. J Cell Physiol. 1997; 173:93-101.

37. Kempen DH, Kruyt MC, Lu L, et al.Effect of Autologous BMSCs Seeding and BMP-2 Delivery on ectopic boneformation in a microsphere/ poly(propylene fumarate) composite. Tissue Eng. Part A 2009;15: 587-94
International Journal of Nanomedicine

\section{Publish your work in this journal}

The International Journal of Nanomedicine is an international, peerreviewed journal focusing on the application of nanotechnology in diagnostics, therapeutics, and drug delivery systems throughout the biomedical field. This journal is indexed on PubMed Central,

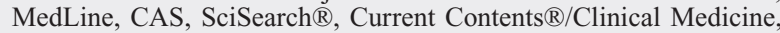

\section{Dovepress}

Journal Citation Reports/Science Edition, EMBase, Scopus and the Elsevier Bibliographic databases. The manuscript management system is completely online and includes a very quick and fair peer-review system, which is all easy to use. Visit http://www.dovepress.com/ testimonials.php to read real quotes from published authors. 